\title{
Spautin-1 Ameliorates Acute Pancreatitis via Inhibiting Impaired Autophagy and Alleviating Calcium Overload
}

\author{
Juan Xiao, ${ }^{1,2}$ Xueping Feng, ${ }^{1}$ Xiao-Ying Huang, ${ }^{2}$ Zhongshi Huang, ${ }^{1,2}$ Yanqiang Huang, ${ }^{1,2}$ Chaogan Li, ${ }^{1}$ \\ Genliang Li, ${ }^{1}$ Song Nong, ${ }^{1}$ Ruoshi Wu, ${ }^{3}$ Yongzhi Huang, ${ }^{3}$ and Xi-Dai Long ${ }^{3,4}$
}

${ }^{1}$ Youjiang Medical University for Nationalities, Baise, People's Republic of China; ${ }^{2}$ Western Guangxi Key Laboratory Cultivation Base of Microbial Infection Research, Baise, People's Republic of China; ${ }^{3}$ Department of Pathology, the Affiliated Hospital of Youjiang Medical University for Nationalities, Baise, People's Republic of China; and ${ }^{4}$ Department of Liver Surgery, Ren Ji Hospital, School of Medicine, Shanghai Jiao Tong University, Shanghai, People's Republic of China

\begin{abstract}
Acute pancreatitis is characterized by zymogen preactivation. Severe inflammation caused by zymogen activation can eventually lead to multiple organ dysfunctions which contribute to the high mortality rate of severe acute pancreatitis. However, there is no specific treatment available for acute pancreatitis therapy. Here, we show that spautin-1, which effectively inhibits autophagy flux, ameliorated the pathogenesis of acute pancreatitis induced by cerulein or L-arginine. CaMKII phosphorylation due to cytosolic calcium overload was revealed in this paper. It was also demonstrated that autophagic protein aggregates degradation blockade accompanied by impaired autophagy correlated positively with intra-acinar cell digestive aymogen activation stimulated by cerulein or L-arginine. The role of spautin- 1 in ameliorating acute pancreatitis was shown here to be associated with impaired autophagy inhibition and $\mathrm{Ca}^{2+}$ overload alleviation. We provide a promising therapy for acute pancreatitis through targeting both impaired autophagy and increased cytosolic calcium.
\end{abstract}

Online address: http://www.molmed.org

doi: $10.2119 / \mathrm{molmed} .2016 .00034$

\section{INTRODUCTION}

Acute pancreatitis is a kind of selflimited disease; about $80 \%$ of patients with acute pancreatitis will recover (1). However, the other $20 \%$ may develop severe acute pancreatitis $(2,3)$. The mortality rate of severe acute pancreatitis is about $30-50 \%$ due to complications such as lung or renal dysfunction $(2,3)$. Among those diverse etiologies, alcohol abuse followed by duct obstruction and bacterial infection induced by a gallstone is the major cause leading to acute pancreatitis (4-7). The incidence of acute pancreatitis is increasing. However, there is no specific treatment available for acute pancreatitis therapy at this time.

Although the mechanism of acute pancreatitis is not fully elucidated, it is believed that early events during the process of acute pancreatitis include vacuole accumulation, calcium overload and cytokine activation $(8,9)$. At the early stage of acute pancreatitis, the

$X F$ and $X-Y H$ should be considered co-second authors.

Address correspondence to Juan Xiao, Youjiang Medical University for Nationalities, Baise 533000, People's Republic of China. Phone: 86-137-68066405; Fax: 0776-2853922; E-mail: xj042386@sina.com; or Xi-Dai Long, Department of Pathology, Affiliated Hospital of Youjiang Medical University for Nationalities, Baise 533000, People's Republic of China. Phone: 86-776-2827667; E-mail: sjtulongxd@263.net.

Submitted February 2, 2016; Accepted for Publication August 11, 2016; Published Online (www.molmed.org) August 18, 2016.

$7 \mathrm{H}$

Feinstein Institute

for Medical Research Northwell Health

digestive zymogens, which normally work after being secreted, are preactivated in pancreatic acinar cells $(10,11)$. Self-digestion as a result causes pancreas injury directly. However, the mechanism underlying the activation of digestive enzymes, especially trypsinogen, is poorly understood.

Autophagy is the process responsible for long-lived protein or organelle degradation. Autophagosome engulfing cargoes fuse with lysosome forming autolysosome where degradation takes place (12-14). Vesicles accumulating in the area of necrotizing pancreatitic tissue were first identified to be autophagosome by electron microscopy (15). Then it was revealed that Atg5 deficiency prevented intracellular trypsinogen from activation in acinar cells, suggesting autophagy as a positive regulator in promoting the activation of digestive zymogens in pancreatic acinar cells (16). Consistently, autophagy flux inhibitor wortmannin was reported to protect against trypsinogen activation induced 
by cerulein and ameliorated the pathogenesis of acute pancreatitis to some extent $(17,18)$. However, wortmannin is not so effective in inhibiting autophagy.

Autophagysome increment resulting in trypsinogen preactivation can be seen in cases of increased autophagy flux or autophagy blockage. It was recently illustrated that autophagysome accumulation due to autophagy blockage is probably the key factor for zymogen activation. In the model of acute pancreatitis induced by alcohol together with lipopolysaccharides (LPS), it was found that the fusion ratio between autophagysome and lysosome decreased due to local lysosome-associated membrane protein 2 (LAMP2) deficiency in inflamed pancreatic tissue (19). Almost at the same time, it was demonstrated that autophagy flux inhibition led to an imbalance between cathepsin B and cathepsin C, which eventually stimulated trypsinogen activation in cerulein-induced acute pancreatitis (20). These results supported the theory that autophagy flux was actually blocked during the process of acute pancreatitis. The concept of impaired autophagy in the pathogenesis of acute pancreatitis was thus established $(20,21)$.

Specific and potent autophagy inhibitor-1 (spautin-1), a well-documented highly effective autophagy inhibitor, was shown to be a valuable therapeutic against viruses through inhibiting autophagy flux $(22,23)$. We hypothesized that spautin-1 decreasing the amount of autophagysome could protect intra-acinar cells from digestive zymogen activation. Here, we tested this hypothesis on the model of acute pancreatitis induced by cerulein or a large dose of L-arginine. Our results indicated that spautin-1 ameliorated cerulein- or L-arginine-induced acute pancreatitis. The mechanism of spautin-1 acting on acute pancreatitis was revealed here to be associated with impaired autophagy inhibition.

Calcium overload, another characteristic of acute pancreatitis, can rapidly activate pancreatic $\mathrm{Ca}^{2+} /$ calmodulin-dependent protein kinase II (CaMKII) under cholecystokinin (CCK) treatment (24). It was indicated that cytosolic calcium and the phosphorylation of CaMKII were up regulated in acute pancreatitis induced by cerulein or L-arginine. Both calcium overload and phosphorylation of CaMKII could be reversed by spautin- 1 .

\section{MATERIALS AND METHODS}

\section{Reagents}

The following commercial antibodies were used: rabbit anti-LC3 (Sigma), rabbit anti-p62 (PTG), rabbit antiubiquitin (PTG), rabbit antitubulin (PTG), rabbit anti-CaMKII $\beta$ (SAB), rabbit anti-phos-CaMKII $\beta$ (PTG), rabbit antibeclin1 (Santa Cruz Biotechnology), rabbit anti-p65 (SAB), rabbit anti-NFkB-p65 (Phospho-Ser536) signalway antibody (SAB) and IgG secondary antibodies (PTG). The following chemical reagents were also used: cerulein (Yeasen), BAPTA-AM (AAT Bioquest), fluo-4-AM (Yeasen), L-arginine and chloriquitone (CQ) (Sigma), butoxycarbonyl-GlnAla-Arg-7-amido-4-methylcoumarin hydrochloride (BGAM) (Sigma), amylase and lipase ELISA Kit (Sigma) and TNF $\alpha$ ELISA Kit (R\&D Systems). Spautin-1 was obtained from the laboratory of Jun Ying Yuan in Shanghai.

\section{Cell Cultures}

The pancreatic cell line AR42J (American Tissue Culture Collection) was cultured in Dulbecco's modified Eagle's medium (Hyclone) containing $10 \%$ fetal bovine serum (Hyclone) and $1 \%$ penicillin-streptomycin (Hyclone) in an atmosphere of $5 \% \mathrm{CO}_{2}$ at $37^{\circ} \mathrm{C}$ using standard techniques.

\section{Animals and Experimental Model}

The study was carried out using 72 female KunMing (KM) mice from Switzerland. The mice were four-weeksold, weighed 20-25g and were obtained from the Shanghai Slac Laboratory Animal Co. All mice were housed in micro-isolator plastic cages and supplied with a basal diet and autoclaved distilled water ad libitum. All animal care and experimental procedures complied with the guidelines for the Care and Use of Laboratory Animals (Eighth Edition), and were approved by the Ethical Committee on Animal Experiments at Youjiang Medical University for Nationalities.

In this study, mice models with acute pancreatitis, including cerulein- and L-arginine-induced models, were constructed as previously described. For the cerulein-induced model, four intraperitoneal injections of cerulein $(50 \mu \mathrm{g} / \mathrm{kg}$ body weight) were given consecutively at hourly intervals (25). The L-arginineinduced model received hourly intraperitoneal injections of $1.4 \mathrm{~g} / \mathrm{kg}$ (optimal dosage for this study) L-arginine three times (26).

Rats were randomly divided into six groups ( $n=12$ per group). The first three groups were designed for ceruleininduced model analysis: Group 1 (control); Group 2 (cerulein), ceruleininduced pancreatitis without spautin-1 treatment; and Group 3 (cerulein + spautin-1), cerulein-induced pancreatitis with spautin- 1 treatment $(2 \mathrm{mg} / \mathrm{kg}$, given by intraperitoneal injection $30 \mathrm{~min}$ before the first cerulein injection). The next 3 groups related to the L-arginine-induced model analysis: Group 4 (control);

Group 5 (L-arginine), L-arginine-induced pancreatitis without spautin-1 treatment; and Group 6 (L-arginine + spautin-1), L-Arginine-induced pancreatitis with spautin- 1 treatment $(2 \mathrm{mg} / \mathrm{kg}$, given by intraperitoneal injection $30 \mathrm{~min}$ before the first L-arginine injection). To observe the changes in the inflammation process, some of the mice were randomly selected and killed for serum amylase analysis three, six and nine hours after the last injection of cerulein or L-arginine. The mice were sacrificed nine hours after the last injection of cerulein or L-arginine for the analysis of serum amylase, lipase and TNF $\alpha$, Western blotting and histopathological change.

\section{Blood Chemistry}

Serum levels of amylase and lipase were determined using the commercial assay kits according to the manufacturer's instructions. 


\section{Histological Analysis}

Pancreatic tissues were obtained from all mice and fixed overnight in 10\% neutral-buffered formalin, routinely paraffin embedded and cut to four $\mathrm{mm}$ for hematoxylin and eosin (H\&E) staining for histological analysis. In this study, histopathological changes, including edema, necrosis, infiltration of inflammation cells and vacuolization were qualified according to the criteria described in Tables 1 and 2 (27). The final score was calculated as the sum of the abovementioned four parameters. All sections were evaluated by two independent pathologists unaware of the study target.

\section{Trypsin Activity Assay}

The lysates from AR42J cells were mixed with BGAM in trypsin reaction buffer $(10 \mathrm{mMmol} /$ LTris, $20 \mathrm{mmol} / \mathrm{L}$ $\mathrm{CaCl}_{2}, \mathrm{pH} 7.4$ ), after 30 min of incubation at $37^{\circ} \mathrm{C}$. The fluorescence intensity was measured at $450 \mathrm{~nm}$ under excitation at $380 \mathrm{~nm}$ (28).

\section{Cytosolic Calcium Analysis}

AR42J cells were loaded with $0.5 \mu \mathrm{mol} / \mathrm{L}$ calcium indicator Fluro-4 AM and the calcium was visualized by fluorescence microscopy imaging (29). Cellular calcium fluorescence intensity was qualified by Image J soft version 1.4.3.67 (National Institutes of Health, United States of America).

\section{Western Blots}

Protein extracts from culture cells and mice pancreatic tissues were separated and quantified by sodium dodecyl sulfate (SDS)-polyacrylamide gel and transferred to the polyvinylidene fluoride (PVDF) membrane (Millipore). After blocking with $5 \%$ nonfat milk in phosphate buffer saline (PBS), membranes were immunoblotted with the indicated primary antibodies (including anti-LC3, anti-p62, antibeclin1, antiubiqutin, anti-phos-p65, anti-NFkb p65, anti-CaMKII and anti-phos-CaMKII bodies), and then with horseradish peroxidase-conjugated immunoglobulin

Table 1. Histopathological change of pancreatic tissues in the mice model with acute pancreatitis induced by cerulein.

\begin{tabular}{lcccc}
\hline Parameter & Group 1 & Group 2 & Group 3 & Pvalue* \\
\hline Edema degree & $1.00 \pm 0.00$ & $3.25 \pm 0.5$ & $2.25 \pm 0.5$ & $<0.001 / 0.03$ \\
Inflammation degree & $0.25 \pm 0.50$ & $2.25 \pm 0.5$ & $1.50 \pm 0.58$ & $0.001 / 0.098$ \\
Vacuolization degree & $0.00 \pm 0.00$ & $2.75 \pm 0.96$ & $2.00 \pm 0.00$ & $0.001 / 0.168$ \\
Necrosis degree & $0.00 \pm 0.00$ & $1.25 \pm 0.50$ & $0.50 \pm 0.58$ & $0.002 / 0.098$ \\
Total score & $1.25 \pm 0.50$ & $9.50 \pm 1.29$ & $6.25 \pm 1.26$ & $<0.001 / 0.011$ \\
\hline
\end{tabular}

*The $P$ value indicates the statistical significance for the differences among Group 1 and 2/among Group 2 and 3.

Group 1: saline control; Group 2: mice injected with cerulein; Group 3: mice injected with cerulein together with spautin-1.

Table 2. Histopathological change of pancreatic tissues in the mice model with acute pancreatitis induced by L-arginine.

\begin{tabular}{lcccc}
\hline Parameter & Group 4 & Group 5 & Group 6 & Pvalue* \\
\hline Edema degree & $1.50 \pm 0.58$ & $3.75 \pm 0.5$ & $2.75 \pm 0.5$ & $0.001 / 0.03$ \\
Inflammation degree & $0.50 \pm 0.58$ & $3.50 \pm 0.58$ & $2.00 \pm 0.82$ & $<0.001 / 0.027$ \\
Vacuolization degree & $0.00 \pm 0.00$ & $2.25 \pm 0.96$ & $2.25 \pm 0.96$ & $0.003 / 1.00$ \\
Necrosis degree & $0.00 \pm 0.00$ & $2.75 \pm 0.50$ & $1.25 \pm 0.50$ & $<0.001 / 0.05$ \\
Total score & $2.00 \pm 0.82$ & $12.25 \pm 2.36$ & $8.25 \pm 0.96$ & $0.002 / 0.035$ \\
\hline
\end{tabular}

*The $P$ value indicates the statistical significance for the differences among Group 4 and 5/among Group 5 and 6.

Group 4: saline control; Group 5: mice injected with L-arginine; Group 6: mice injected with L-arginine together with spautin-1.

G (IgG) secondary antibodies for two hours at room temperature. In this study, anti- $\alpha$-tubulin antibody acted as the internal control. The resulting bands were visualized with an electrochemiluminescence (ECL) system (Amersham Biosciences).

\section{The Ubiquitination Analysis of Protein Aggregates}

In this study, ubiquitination (Ub) levels of protein aggregates were elucidated by the above-mentioned Western blotting. Briefly, 1\% Triton X-100-soluble or insoluble fractions from culture cells and mice pancreatic tissues were dissolved in $2 \%$ SDS separately, and then were analyzed by immunoblotting with antiubiquitin antibody.

\section{Statistical Analysis}

Student $t$ test or one-way ANOVA were used to analyze the significance of the differences between groups. Error bars for microscopy were presented as the standard deviation of triplicate samples. Error bars for Western blot analysis represent the standard deviation between densitometry data from three unique experiments. All statistical analyses were done using the statistical package for social science (SPSS) version 18 (SPSS Institute).

All supplementary materials are available online at www.molmed.org.

\section{RESULTS}

\section{Cerulein or L-Arginine Inhibiting Autophagy Flux Correlates with Trypsinogen Activation in Pancreatic Acinar Cells}

Previous reports have shown that animal models with acute pancreatitis can be produced by cerulein or a large dose of L-arginine (30). Autophagy is impaired and this damage can promote trypsinogen activation (31). Thus, we questioned whether this cerulein- or L-arginineinduced autophagy damage arose in vitro. We treated acinar cells AR42J with cerulein (Figure 1A) in the presence or absence of chloriquitone (CQ) which can block 
A
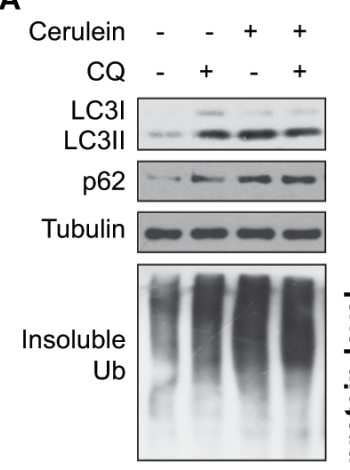

Soluble

Ub

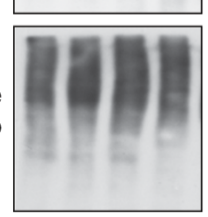

C

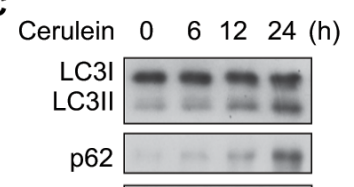

Beclin1 $-\cdots-$
Tubulin $=-\infty$
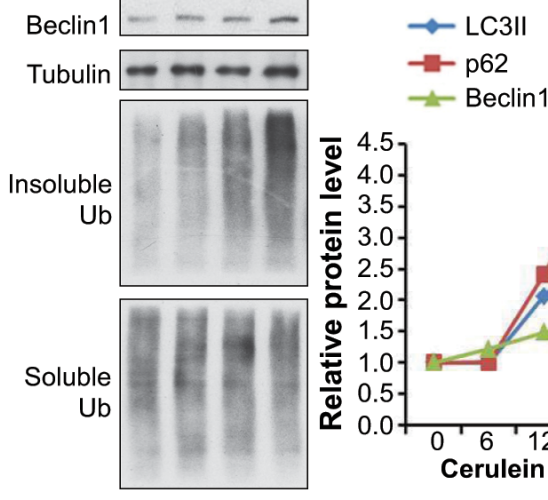

E

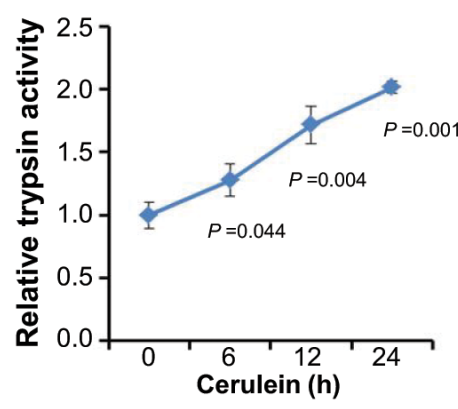

B
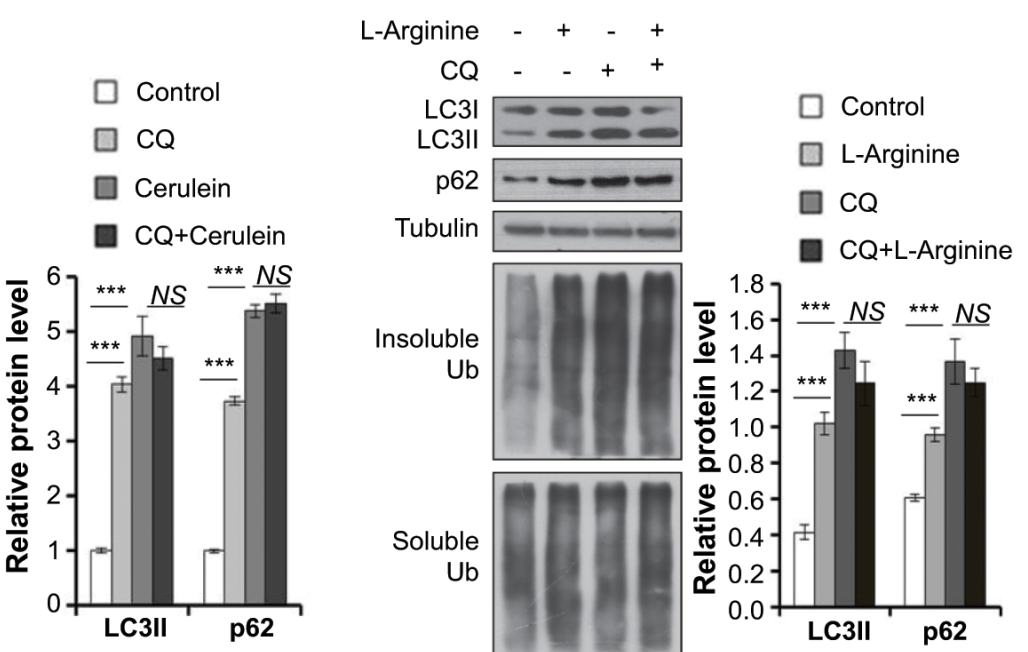

D

L-Arginine $\quad 0 \quad 6 \quad 12 \quad 24(h)$
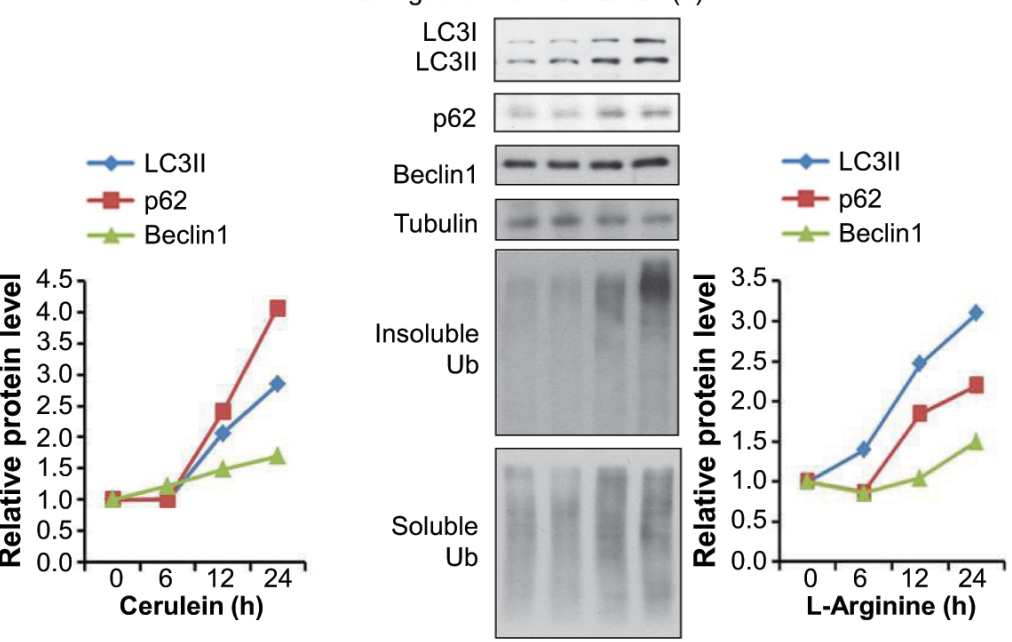

$\mathbf{F}$

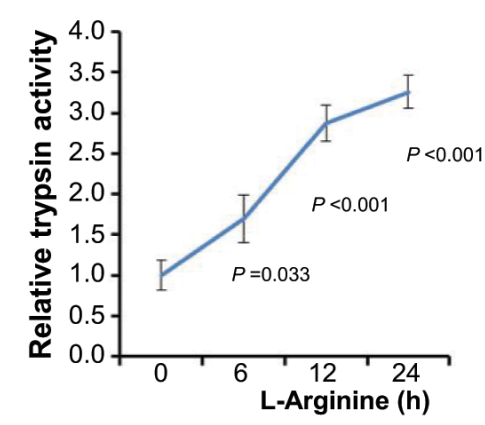

Figure 1. Cerulein or L-arginine inhibiting autophagy flux correlates with trypsinogen activation in pancreatic acinar cells. (A, B). AR42J cells were treated with $200 \mathrm{nmol} / \mathrm{L}$ cerulein (A) or $10 \mathrm{mmol} / \mathrm{L}$ L-arginine (B) for $22 \mathrm{~h}$ followed by chloriquitone (CQ) or normal saline treatment (Control) for two hours. The levels of LC3II, p62 and ubiquitination (Ub) were next analyzed using the Western blotting technique. The graphs provided are quantified using Image $J$ as means \pm SD of three independent sets of experiments. Student $t$ test was used. ${ }^{* * *} P<0.001$. (C-F) AR42J cells were treated with $200 \mathrm{nmol} / \mathrm{L}$ cerulein $(C, E)$ or $10 \mathrm{mmol} / \mathrm{L} L$-arginine $(D, F)$ for indicated durations. The levels of $L C 3$, $p 62$, beclin 1 and $\mathrm{Ub}$ were next analyzed using the Western blotting technique (C, D), and the corresponding graphs (right line graphs) were quantified using Image $\mathrm{J}$ from two independent sets of experiments (C, D). Error bars represent SD. The relative trypsin activity was evaluated by the fluorescence intensity. Student $t$ test was used as statistical analysis from three independent sets of experiments (E, F). 
the fusion between autophagosome and lysosome. Results showed that the level of microtubule-associated protein light chain 3 form II (LC3II) (a marker of autophagy) increased when cells were treated with only cerulein or CQ (Figure 1A). However, there were no further increments in LC3II level under the conditions of treatment with both cerulein and CQ (Figure 1A). This suggests that autophagy could be blocked in pancreatic acinar cells under cerulein treatment. Similar results were obtained for L-arginine-treated pancreatic acinar cells (Figure 1B).

To further elucidate the role of autophagy in pancreatic acinar cells with cerulein or L-arginine treatment, we next detected the protein level of p62 (also known as SQSTM), an important scaffolding protein involving in the ubiquitination degradation of protein aggregations in autolysosome (32). Consistent with the increased level of LC3II, the level of p62 gradually elevated with the time and dose of cerulein treatment (Figure 1C and Supplementary Figure S1A) or L-arginine treatment (Figure 1D and Supplementary Figure S1B). Since ubiquitylated protein aggregates are transported into autophagysome for degradation by p62, we also investigated the effect of cerulein or L-arginine on the ubiquitination of protein aggregates. Results from Western blotting revealed that ubiquitination of protein aggregates was upregulated significantly in insoluble fraction; while soluble ubiquitination remained almost constant (Figures 1A-D; Supplementary Figures S1A, S1B). Compared to LC3II, p62 and ubiquitylated protein aggregates, the level of Beclin1 expression (an autophagy flux positive regulator) slightly increased (Figures 1C, D; Supplementary Figures S1A, S1B). To further confirm the relationship between impaired autophagy and the pathogenesis of acute pancreatitis, we performed an in vitro reaction catalyzed by trypsin from pancreatic acinar cells. It was found that trypsin activity in acinar cells gradually increased with increasing levels of LC3II and p62 (Figures 1E, F; Supplementary Figures S1C, S1D). In summary, these results suggested that autophagy flux inhibition induced by cerulein or L-arginine might correlate with trypsinogen activation in vitro.

Taken together, we concluded that autophagy flux blockade also referred to impaired autophagy in pancreatic acinar cells upon cerulein or L-arginine treatment. Our study supported the theory that impaired autophagy was important for trypsinogen activation in the process of acute pancreatitis.

\section{Spautin-1 Reduced Impaired Autophagy and Inhibited Trypsinogen Activation in Pancreatic Acinar Cells with Cerulein or L-Arginine Treatment}

Because spautin-1 has potential therapeutic effects in antivirus infection or cancer therapy due to its high efficiency in autophagy flux inhibition $(23,33)$, we hypothesized that spautin-1 could protect against trypsinogen activation. To prove this, we determined whether trypsin activity induced by cerulein or L-arginine in the acinar cells AR42J decreased under the condition of spautin-1 treatment (Figures 2A, B). The results showed that spautin-1 can inhibit trypsinogen activation induced by cerulein (Figure 2A) or L-arginine (Figure 2B).

To determine the relationship between spautin-1 inhibiting trypsinogen activation and impaired autophagy in intra-acinar cells, we detected protein levels of LC3II, p62 and ubiquitylated protein aggregates using Western blotting (Figure 2C-F). The results showed that the levels of both LC3II and p62 decreased in the AR42J cells with both cerulein and spautin-1 treatment compared with cerulein but without spautin-1 treatment (Figures 2C, D). Moreover, this downregulation by spautin-1 occurs in a time-dependent manner (Figure 2D). Of note, the levels of ubiquitylated protein aggregates decreased in insoluble fraction but increased in soluble ubiquitination under spautin-1 treatment, which is consistent with the function of spautin-1 promoting ubiquitination (Figures 2C, D). Similar results were also obtained with cells in the case of L-arginine treatment (Figures 2E, F). These data suggest that spautin-1 protecting against trypsinogen activation in intraacinar cells may be partly ascribed to its function in inhibiting impaired autophagy.

\section{Spautin-1 Ameliorated Acute Pancreatitis in Mice}

To elucidate the therapeutic potential of spautin- 1 for acute pancreatitis, we constructed mice models with acute pancreatitis using cerulein or L-arginine and explored the therapeutic role of spautin-1 in acute pancreatitis in these models. In the cerulein-induced acute pancreatitis model, we observed that spautin- 1 pretreatment significantly diminished the elevation of serum amylase and lipase levels, which are indicative of trypsin activity (Figure 3A left and middle). Given that pancreas injury from trypsinogen preactivation was usually accompanied by an inflammation reaction, we next detected the effects of spautin- 1 on inflammation indicators tumor necrosis factor $\alpha$ (TNF $\alpha)$ and p65 (the subunit of NFא $\beta$ ) (Figures 3A right and $3 \mathrm{~B}$ ). Interestingly, increasing levels of serum TNF $\alpha$ caused by cerulein were inhibited in the presence of spautin-1 (Figure 3A right). For p65, although its expression was little changed in the pancreas tissues from mice with acute pancreatitis, its active form, phos-p65, was downregulated by spautin-1 (Figure 3B). Histopathological analyses further showed that the spautin-1 treatment can ameliorate the inflammation damage induced by cerulein, such as edema, degeneration, coagulative necrosis and infiltration of inflammatory cells (Table 1). Representative graphs exhibited this kind of damage (Figure 3C).

Since spautin- 1 has been found to decrease impaired autophagy in vitro, we further detected the levels of LC3II, p62 and beclin 1 protein in vivo. Results showed that increasing levels of p62 and beclin1 protein in pancreatic tissues with acute inflammation decrease under the condition of spautin-1 treatment (Figure 3D). Correspondingly, the elevation of ubiquitylated protein aggregates in insoluble fraction diminish and the level of ubiquitination in soluble fraction increases (Figure 3D). 
A

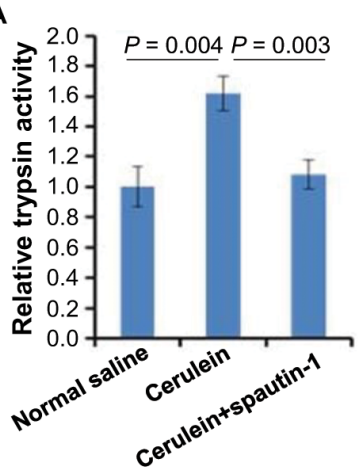

C

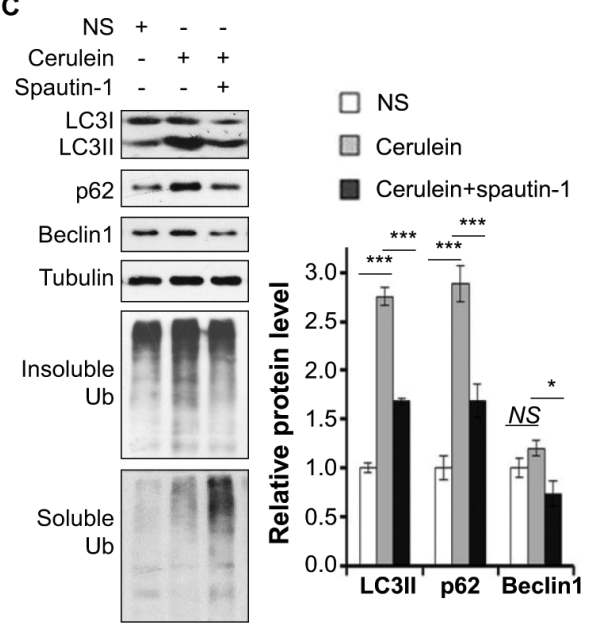

B

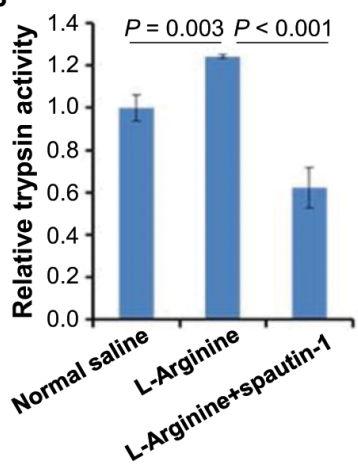

D

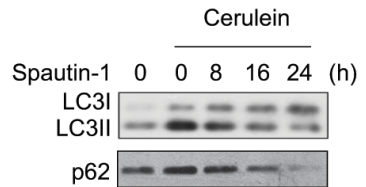

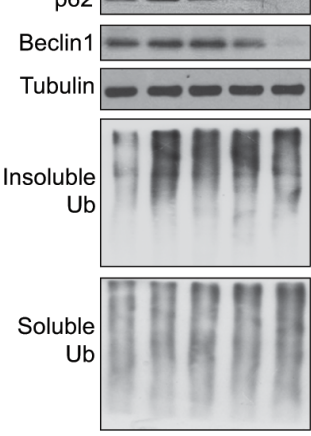

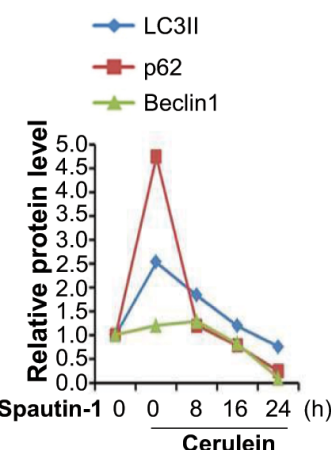

E

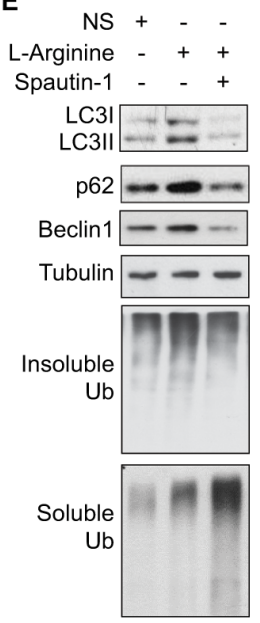

$\mathbf{F}$

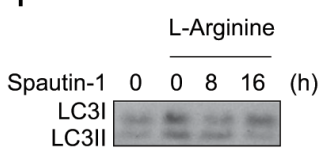

(h)
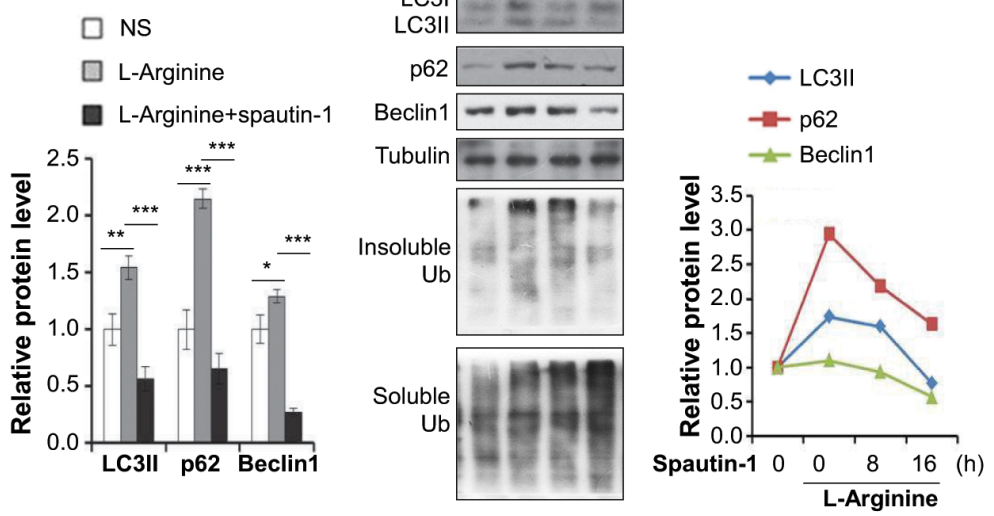

Figure 2. Spautin-1 reduces impaired autophagy and inhibited trypsinogen activation in pancreatic acinar cells with cerulein or L-arginine treatment. (A, B) AR42J cells were treated with normal saline (NS), $200 \mathrm{nmol} / \mathrm{L}$ cerulein (A) or $10 \mathrm{mmol} / \mathrm{L} \mathrm{L}-$ arginine (B) in the presence or absence of $10 \mu \mathrm{mol} / \mathrm{L}$ spautin- 1 for $24 \mathrm{~h}$. The trypsin activity was next analyzed by the fluorescence intensity. Data from three independent experiments were tested Student $t$ test and shown as means \pm SD. (C-F) AR42J cells with indicated treatment were lysed and were analyzed by Western blotting with anti-LC3, anti-p62, antibeclin 1, Ub and antitubulin antibodies. The relative levels of LC3II, p62 and beclin 1 expression were are quantified using Image $\mathrm{J}$ and exhibited as the bar graphs from three independent sets of experiments (C, E) and line graphs from two independent sets of experiments (D, F). Error bars represent SD. Student $t$ test was used ${ }^{*} P<0.05,{ }^{* *} P<0.01,{ }^{* * *} P<0.001$. 

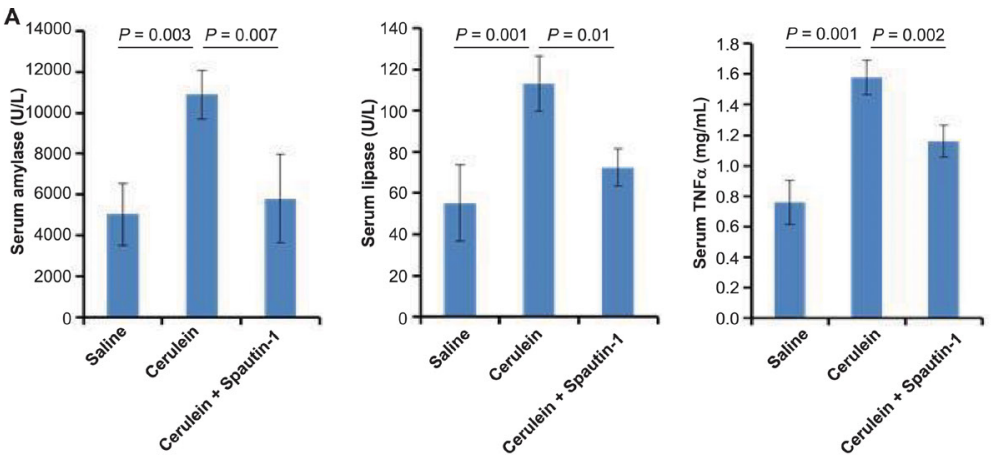

$$
\text { B }
$$
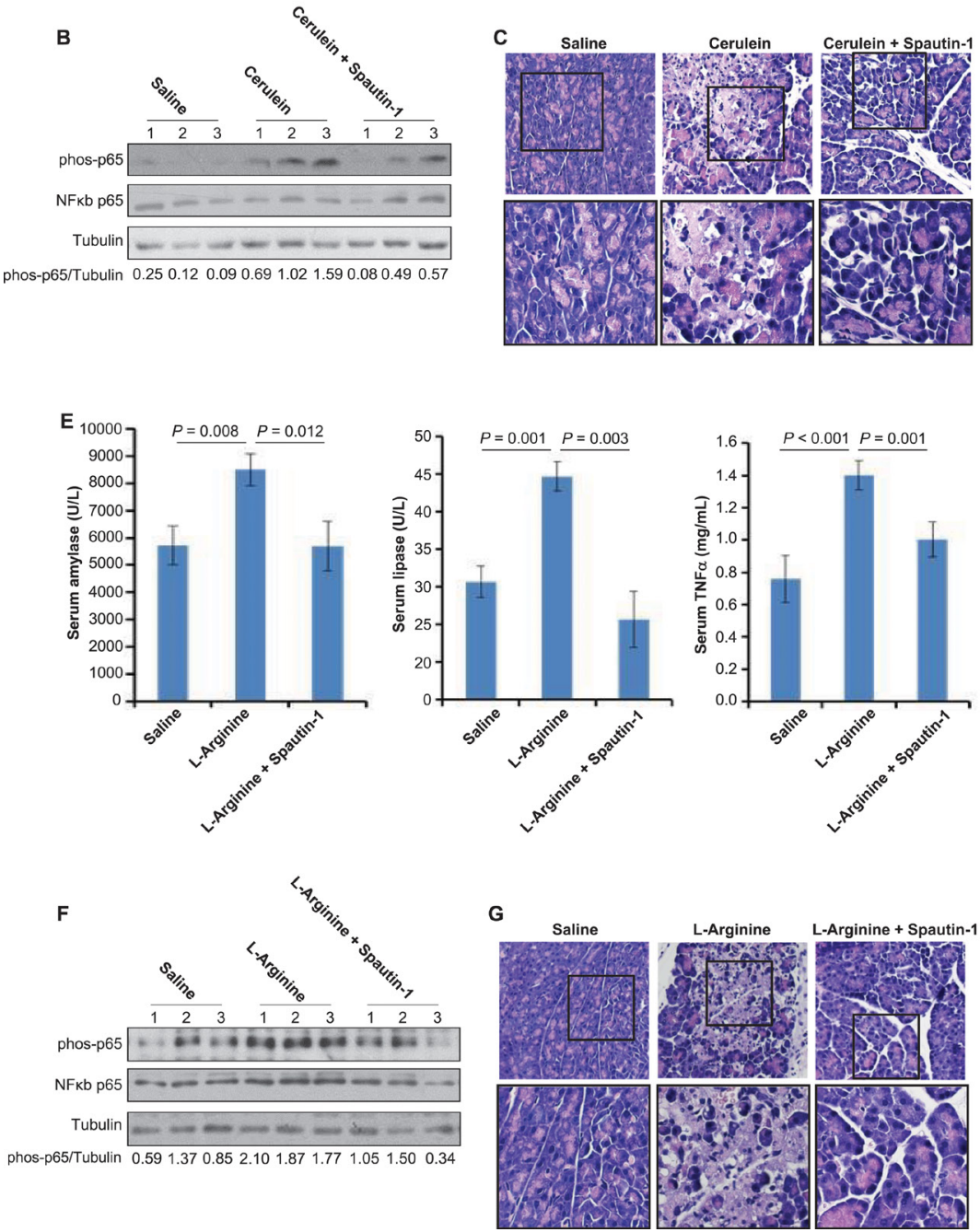
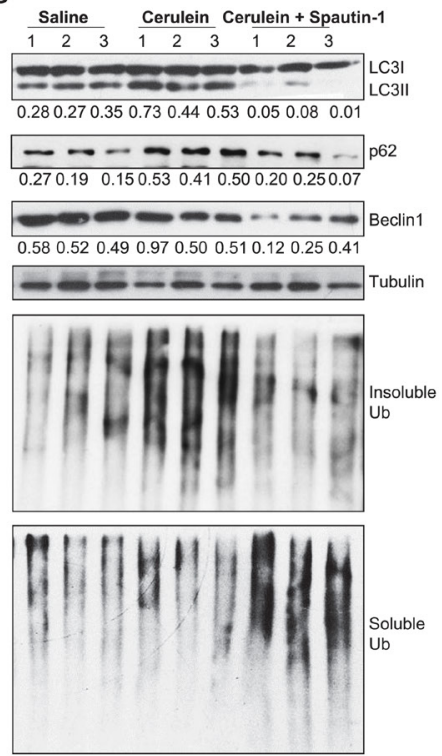

$\mathrm{H}$
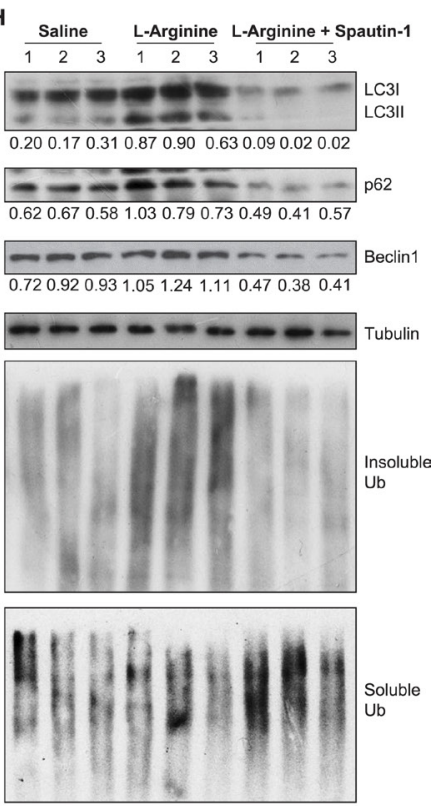

Figure 3. Spautin-1 ameliorates the pathogenesis of acute pancreatitis induced by cerulein or L-arginine in mice. (A-D) Cerulein-induced mice model with acute pancreatitis. Spautin-1 was injected half an hour before the first injection of cerulein. The mice were sacrificed nine hours after the last cerulein injection for analyzing serum amylase, lipase and TNFa levels (A), histopathological change (C) and the levels of p65 (B), LC3II, p62, beclin 1 and Ub (D) in the pancreatic tissues. The data from serum analyses were tested using one-way ANOVA with LSD corrections and shown as means \pm SD. $(E-H)$ L-arginine-induced mice model with acute pancreatitis. Spautin- 1 was injected $30 \mathrm{~min}$ before the first injection of L-arginine. The mice were sacrificed nine hours after the last L-arginine injection for analyzing serum amylase, lipase and TNFa levels (E), histopathological change (G) and the levels of p65 (F), LC3II, p62, beclin 1 and Ub (H) in the pancreatic tissues. The data from serum analyses were tested using one-way ANOVA with LSD corrections and shown as means \pm SD. The ratios between the level of relative protein and tubulin are listed below the band. 
Similar results were observed in the mice model with acute pancreatitis using L-arginine (Table 2, Figure 3E-H). Taken together, these data suggest that spautin-1 should have therapeutic potential for acute pancreatitis, possibly through inhibiting impaired autophagy.

\section{Spautin-1 Reversed Cytosolic Calcium Overload Induced by Cerulein or L-Arginine In Vitro and In Vivo}

Calcium overload is a well-known characteristic of acute pancreatitis. Here, we detected cytosolic calcium using calcium sensitive dye fluo-4-AM and found that cytosolic calcium was elevated in ceruleinor L-arginine-treated AR42J cells in vitro (Figures 4A, B). Because calcium overload can result in trypsinogen preactivation in acute pancreatitis (34), calcium chelator BAPTA-AM was used to conform the relationship between calcium elevation and trypsinogen activation in the present study. Correspondingly, trypsinogen activation in acinar cells was alleviated under the conditions of BAPTA-AM (Figures 4C, D).

Given that cytosolic calcium may affect autophagy (35-37), we questioned whether spautin-1 played a role in the calcium signaling pathway. To answer this, we first evaluated fluorescence intensity in pancreatic acinar cells with or without spautin-1 treatment and found spautin-1 significantly diminished cytosolic calcium load caused by cerulein or L-arginine (Figures 4E, F). We next analyzed the changes of CaMKII (including its phosphorylation form) in pancreatic acinar cells treated with cerulein or L-arginine. Results showed that the phosphorylation of CaMKII gradually increased with the time and dose of cerulein or L-arginine treatment, but the level of CaMKII changed only slightly (Figures 4G, $\mathrm{H}$; Supplementary Figures S3A, S3B). Interestingly, this increasing phosphorylation level induced by cerulein or L-arginine would decrease under the conditions of spautin-1 treatment (Figure 4I, J). Together, these data further supported that spautin-1 ameliorating the pathogenesis of acute pancreatitis might be correlated with relieving calcium overload.

\section{DISCUSSION}

Here, we reported that spautin-1 effectively protected against trypsinogen activation induced by cerulein or L-arginine in vitro or in vivo and ameliorated acute pancreatitis. This is likely mediated via decreasing the level of impaired autophagy and alleviating cytosolic calcium overload.

Autophagic vesicle accumulation is an important event during the early process of acute pancreatitis $(8,9)$. LC3II as indicative of autophagy located in the inner membrane of the autolysosome. Autophagy flux elevation leading to increased LC3II production or autophagy blockade leading to decreased fusing between LC3II and lysosome can upregulate the level of LC3II. Our results indicated that the increased level of LC3II in pancreatic acinar cells upon cerulein or L-arginine treatment was mainly due to the inhibition of autophagy flux. Another evidence for impaired autophagy in pancreatitis is that the change of autophagy flux positive regulator beclin1 is minor. It needs to be mentioned that LC3I shared a similar trend as LC3II under cerulein or L-arginine treatment. We can easily understand the fluctuations of LC3II, which is the actual autophagy indicator for most cases. LC3I can be upregulated when autophagy is blocked (38), hence it sharing a similar trend as LC3II. The functioning of autophagy independently of LC3I has also been revealed $(39,40)$. Thus some unknown events may exist under cerulein or L-arginine treatment.

Protein aggregates were revealed here to correlate with trypsin activity. Ubiquitylated protein aggregates degraded by autophagy may directly trigger the activation of trypsinogen possibly causing cellular toxicity $(41,42)$. Spautin-1 was shown here to promote the transformation of ubiquitylated protein aggregates to soluble form which was accompanied with trypsin activation. Thus protein aggregate accumulation as the result of autophagy blockade might be an important target in ameliorating acute pancreatitis induced by cerulein or L-arginine.
Meanwhile, there still exists evidence suggesting that autophagy promoted pancreatic acinar cells survival. Autophagy responsible for engulfing zymogen is referred to zymophagy, which was inhibited by 3-MA leading to acinar cell death (28). Besides, more trypsin activity was found in Atg5ko mice recently (43). Taken together, these two seemingly controversial evidence suggest the hypothesis put forward here, that damage in pancreatic tissue inducing autophagy which eliminates the protein or organelle with injury benefits cells survival at the beginning, when the damage is supra the extent that the system can stand, impaired autophagy occurs which causes the activation of trypsinogen. In the previous research (16) Atg5 had not been fully knocked out, thus autophagy still existed in Atg5ko mice. In this case, damage still could be eliminated at the early stage of pancreatitis but a lower level of impaired autophagy was induced at the later stage which resulted in a decreased level of trypsinogen activation. However, autophagy was largely inhibited in mice with high deficiency of Atg5 resulting in damage accumulation from the beginning, which induced more trypsinogen activation (43). Thus whether autophagy is beneficial or not depends on the phases of the acute pancreatitis early stage. This hypothesis is still under exploration.

Cytosolic calcium has been recently reported to have an impact on the level of impaired autophagy in liver injury induced by a high fat diet (HFD). In that case, autophosphorylation of CaMKII, which is indicative of an elevated level of cytosolic calcium in hepatocytes, was induced. Correspondingly, calcium channel blocker verapamil could alleviate liver injury (29). Thus it is reasonable that spautin-1 ameliorated the pathogenesis of acute pancreatitis partly due to alleviating calcium overload.

Cytosolic calcium elevation leads to impaired autophagy accumulation in the case of liver injury caused by HFD 
A
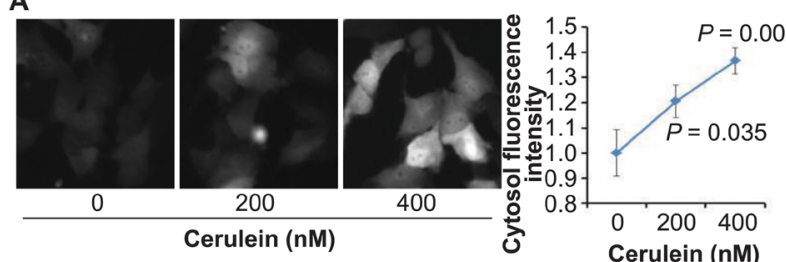

Cerulein (nM)
E

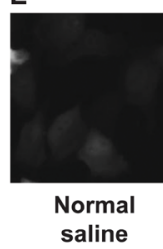

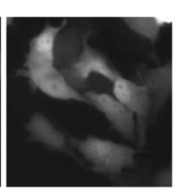

Cerulein

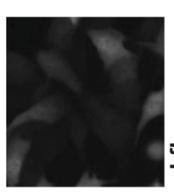

Cerulein +spautin-1

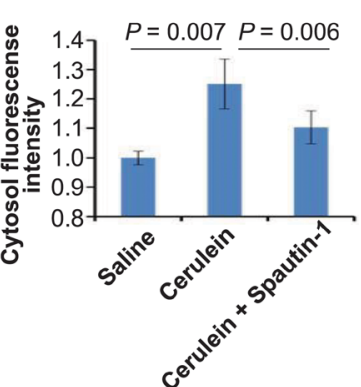

$\mathbf{F}$

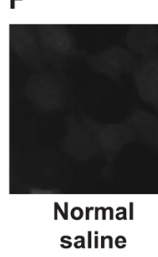

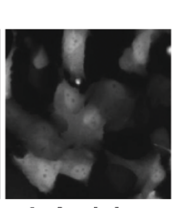

L-Arginine

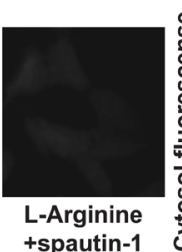

B
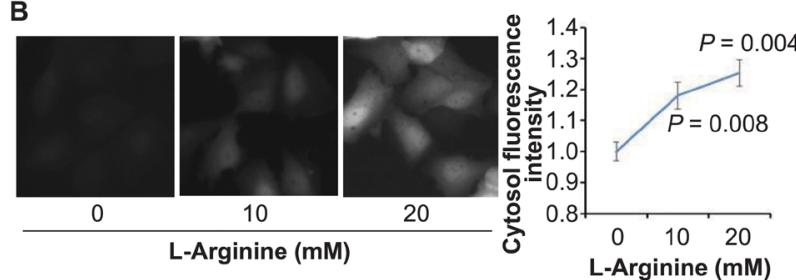

G

Cerulein

p-CaMKII $\beta$

CaMKII $\beta$

Tubulin
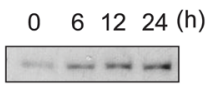

$-\cdots-$

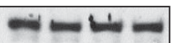

H

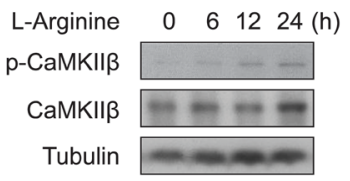

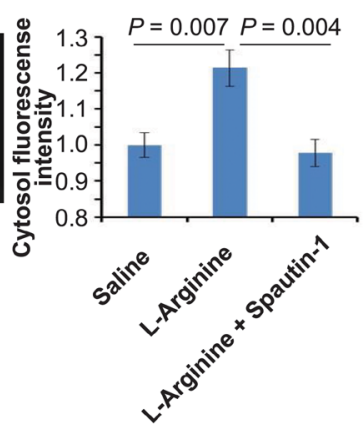

I

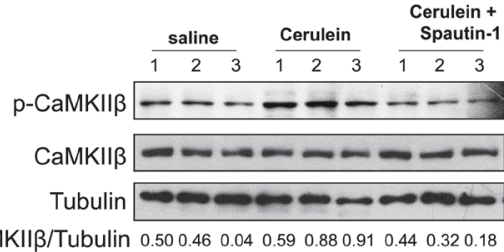

P-CAMKIII/Tubulin 0.500 .460 .040 .590 .880 .910 .440 .320 .18

J

saline L-Arginine $\begin{array}{r}\text { L-Arginine * } \\ \text { Spautin-1 }\end{array}$

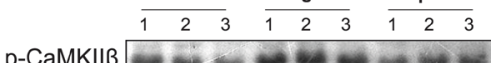
СаMKII $=-\ldots-\ldots \ldots-\ldots$

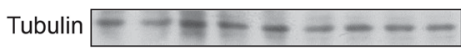
$\begin{array}{llllllllll}\text { P-CAMKIIß/Tubulin } & 2.1 & 2.0 & 1.7 & 2.4 & 2.5 & 2.5 & 1.5 & 1.8 & 1.7\end{array}$

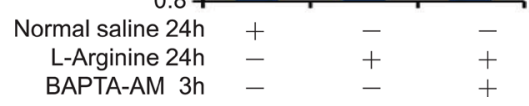

Figure 4. Spautin-1 reverses cytosolic calcium overload induced by cerulein or L-arginine in vitro and in vivo. (A, B) AR42J cells were treated with the indicated concentration of cerulein (A) or L-arginine (B) for $24 \mathrm{~h}$ and then cells were stained with Fluo-4-AM according to manufacturer's instructions. The fluorescence images were analyzed by Image J. The graphs provided are quantified using Image $\mathrm{J}$ as means \pm SD of three independent sets of experiments. Student t test was used. (C, D) AR42J cells were treated with normal saline, $200 \mathrm{nmol} / \mathrm{L}$ cerulein (C) or $10 \mathrm{mmol} / \mathrm{L} \mathrm{L}$-arginine (D) for $21 \mathrm{~h}$ and were then in the presence or absence of $2 \mu \mathrm{mol} / \mathrm{L}$ BAPTA-AM for an additional three hours. The trypsin activity was next analyzed by the fluorescence intensity. Data from three independent experiments were tested with Student $t$ test and shown as means \pm SD. (E, F) AR42J cells were treated with $200 \mathrm{nmol} / \mathrm{L}$ cerulein (E) or $10 \mathrm{mmol} / \mathrm{L} L$-arginine (F) for $24 \mathrm{~h}$ in the presence or absence of $10 \mu \mathrm{mol} / \mathrm{L}$ spautin-1. After that cells were stained with Fluo-4-AM according to manufacturer's instructions, the fluorescence images were analyzed by image $\mathrm{J}$. The graphs provided are quantified using Image $\mathrm{J}$ as means $\pm \mathrm{SD}$ of three independent sets of experiments. Student $t$ test was used. Lysates from AR42J cells $(G, H)$ or mice pancreas $(I, J)$ with indicated treatment were analyzed by Western blotting with anti-CaMKII, anti-phos-CaMKII and antitubulin antibodies.

which could be reversed by verapamil (29). Thus impaired autophagy existed in cerulein- or L-arginine-induced acute pancreatitis may partly due to calcium overload. Similar to the indicative role of calcium in hepatocyte, the phosphorylation of CaMKII could be considered to be a marker of an increased calcium level in pancreatic acinar cells. 


\section{CONCLUSION}

Although spautin-1 was shown to be toxic in long-term treatment (23), it could effectively inhibit impaired autophagy and reverse the increased level of cytosolic calcium. In summary, we have provided a promising therapy for acute pancreatitis through targeting both impaired autophagy and increased cytosolic calcium.

\section{DISCLOSURE}

This study was supported in part by the National Natural Science Foundation of China (No. 81572353, 81372639, 81160255, 81472243 , and 81460423), the Innovation Program of Guangxi Municipal Education Department (No. 201204LX674), Innovation Program of Shanghai Municipal Education Commission (No.13YZ035), the Natural Science Foundation of Guangxi (No. 2015GXNSFAA139223, 2013GXNSFAA019251, 2014GXNSFDA118021, and 2014GXNSFAA118144), Key Discipline and Specialty Foundation of Shanghai Municipal Commission of Health and Family Planning, "Shu Guang" project (supported by Shanghai Municipal Education Commission and Shanghai Education Development Foundation, NO. 13SG19) and the Recruitment Program of Youjiang Medical University for Nationality (No. yy2015hsky03).

\section{REFERENCES}

1. Carroll JK, Herrick B, Gipson T, Lee SP. (2007) Acute pancreatitis: diagnosis, prognosis, and treatment. Am. Fam. Physician. 75:1513-20.

2. Johnson CD, Abu-Hilal M. (2004). Persistent organ failure during the first week as a marker of fatal outcome in acute pancreatitis. Gut. 53:1340-4.

3. Mofidi R, et al. (2006). Association between early systemic inflammatory response, severity of multiorgan dysfunction and death in acute pancreatitis. Br. J. Surg. 93:738-44.

4. Frossard JL, Steer ML, Pastor CM. (2008). Acute pancreatitis. Lancet. 371:143-152.

5. Apte MV, Pirola RC, Wilson JS. (2010). Mechanisms of alcoholic pancreatitis. J. Gastroenterol. Hepatol. 25:1816-26.

6. Harper SJ, Cheslyn-Curtis S. (2011). Acute pancreatitis. Ann. Clin. Chem. 48:23-37.

7. Braganza JM, Lee SH, McCloy RF, McMahon MJ. (2011). Chronic pancreatitis. Lancet. 377:1184-97.

8. Mitchell RM, Byrne MF, Baillie J. (2003). Pancreatitis. Lancet. 361:1447-55.
9. Oiva J, et al. (2010). Acute pancreatitis with organ dysfunction associates with abnormal blood lymphocyte signaling: controlled laboratory study. Crit. Care. 14:R207.

10. Gorelick FS, Matovcik LM. (1995). Lysosomal enzymes and pancreatitis. Gastroenterology. 109:620-5.

11. Halangk W, Lerch MM. (2005). Early events in acute pancreatitis. Clin. Lab. Med. 25:1-15.

12. Xie Z, Klionsky DJ. (2007). Autophagosome formation: core machinery and adaptations. Nat. Cell Biol. 9:1102-9.

13. Mizushima N, Levine B, Cuervo AM, Klionsky DJ. (2008). Autophagy fights disease through cellular self-digestion. Nature. 451:1069-75.

14. Levine B, Kroemer G. (2008). Autophagy in the pathogenesis of disease. Cell. 132:27-42.

15. Helin H, Mero M, Markkula H, Helin M. (1980). Pancreatic acinar ultrastructure in human acute pancreatitis. Virchows Arch. A, Pathol. Anat. Histol. 387:259-70

16. Hashimoto D, et al. (2008). Involvement of autophagy in trypsinogen activation within the pancreatic acinar cells. J. Cell Biol. 181:1065-72.

17. Singh VP, et al. (2001). Phosphatidylinositol 3-kinase-dependent activation of trypsinogen modulates the severity of acute pancreatitis. J. Clin. Invest. 108:1387-95.

18. Xu P, Wang J, Yang ZW, Lou XL, Chen C. (2013). Regulatory roles of the PI3K/Akt signaling pathway in rats with severe acute pancreatitis. PLoS One. 8:e81767.

19. Fortunato F, Kroemer G. (2009). Impaired autophagosome-lysosome fusion in the pathogenesis of pancreatitis. Autophagy. 5:850-3.

20. Mareninova OA, et al. (2009). Impaired autophagic flux mediates acinar cell vacuole formation and trypsinogen activation in rodent models of acute pancreatitis. J. Clin. Invest. 119:3340-55.

21. Gukovsky I, Gukovskaya AS. (2010). Impaired autophagy underlies key pathological responses of acute pancreatitis. Autophagy. 6:428-9.

22. Liu J, et al. (2011). Beclin1 controls the levels of p53 by regulating the deubiquitination activity of USP10 and USP13. Cell. 147:223-34.

23. Mateo R. (2013). Inhibition of cellular autophagy deranges dengue virion maturation. J. Virol. 87:1312-21.

24. Duan RD, Guo YJ, Williams JA. (1994). Conversion to $\mathrm{Ca}(2+)$-independent form of $\mathrm{Ca} 2+/$ calmodulin protein kinase II in rat pancreatic acini. Biochem. Biophys. Res. Commun. 199:368-73.

25. Jacob TG, Raghav R, Kumar A, Garg PK, Roy TS. (2014). Duration of injury correlates with necrosis in caerulein-induced experimental acute pancreatitis: implications for pathophysiology. Int. J. Exp. Pathol. 95:199-208.

26. Kui B, et al. (2015). New insights into the methodology of L-arginine-induced acute pancreatitis. PLoS One. 10:e117588.

27. Yuan W, et al. (2015). E-cadherin expression in a rat model of acute pancreatitis. Exp. Ther. Med. 10:2088-92

28. Grasso D, et al. (2011). Zymophagy, a novel selective autophagy pathway mediated by
VMP1-USP9x-p62, prevents pancreatic cell death. J. Biol. Chem. 286:8308-24.

29. Park HW, et al. (2014). Pharmacological correction of obesity-induced autophagy arrest using calcium channel blockers. Nat. Commun. 5:4834

30. Ma X, et al. (2015). Rise and fall of subclones from diagnosis to relapse in pediatric B-acute lymphoblastic leukaemia. Nat. Commun. 6:6604.

31. Merza M, et al. (2015). Neutrophil extracellular traps induce trypsin activation, inflammation, and tissue damage in mice with severe acute pancreatitis. Gastroenterology. 149:1920-31 e1928.

32. Pankiv S, et al. (2007). p62/SQSTM1 binds directly to Atg8/LC3 to facilitate degradation of ubiquitinated protein aggregates by autophagy. J. Biol. Chem. 282:24131-45.

33. Tong XP, et al. (2015). Key autophagic targets and relevant small-molecule compounds in cancer therapy. Cell Prolif. 48:7-16.

34. Raraty M, et al. (2000). Calcium-dependent enzyme activation and vacuole formation in the apical granular region of pancreatic acinar cells. Proc. Natl. Acad. Sci. U. S. A. 97:13126-31.

35. Hoyer-Hansen M, et al. (2007). Control of macroautophagy by calcium, calmodulin-dependent kinase kinase-beta, and Bcl-2. Mol. Cell. 25:193-205.

36. Williams A, et al. (2008). Novel targets for Huntington's disease in an mTOR-independent autophagy pathway. Nat. Chem. Biol. 4:295-305.

37. Xia HG, et al. (2010). Control of basal autophagy by calpain1 mediated cleavage of ATG5. Autophagy. 6:61-6.

38. Klionsky DJ, et al. (2016). Guidelines for the use and interpretation of assays for monitoring autophagy (3rd edition). Autophagy. 12:1-222.

39. Cottam EM, et al. (2011). Coronavirus nsp6 proteins generate autophagosomes from the endoplasmic reticulum via an omegasome intermediate. Autophagy. 7:1335-47.

40. Reggiori F, et al. (2010). Coronaviruses Hijack the LC3-I-positive EDEMosomes, ER-derived vesicles exporting short-lived ERAD regulators, for replication. Cell Host Microbe. 7:500-8.

41. Wang Z, Figueiredo-Pereira ME. (2005). Inhibition of sequestosome 1/p62 up-regulation prevents aggregation of ubiquitinated proteins induced by prostaglandin $\mathrm{J} 2$ without reducing its neurotoxicity. Mol. Cell. Neurosci. 29:222-31.

42. Grenier C, Bissonnette C, Volkov L, Roucou X. (2006). Molecular morphology and toxicity of cytoplasmic prion protein aggregates in neuronal and non-neuronal cells. J. Neurochem. 97:1456-66.

43. Diakopoulos KN, et al. (2015). Impaired autophagy induces chronic atrophic pancreatitis in mice via sex- and nutrition-dependent processes. Gastroenterology. 148:626-38 e617.

Cite this article as: Xiao J, et al. (2016) Spautin-1 ameliorates acute pancreatitis via inhibiting impaired autophagy and alleviating calcium overload. Mol. Med. 22:643-52. 